\title{
Identification of a novel alternative splicing transcript variant of the suppressor of fused: Relationship with lymph node metastasis in pancreatic ductal adenocarcinoma
}

\author{
QING XU, JUN GAO and ZHAOSHEN LI
}

Department of Gastroenterology in Changhai Hospital, Second Military Medical University, Shanghai 200433, P.R. China

Received August 8, 2016; Accepted October 19, 2016

DOI: 10.3892/ijo.2016.3753

\begin{abstract}
Pancreatic ductal adenocarcinoma (PDAC) is one of the most fatal diseases, and the median survival time is very short. Upregulation of hedgehog signaling pathway activity is a vital factor in pathogenesis of PDAC. However, as a negative regulator of hedgehog signaling, it is not very clear what role the suppressor of fused (SUFU) plays in PDAC tissue. In our study for the identification of alternative splicing transcripts of $S U F U$ gene in human PDAC cells, a novel transcript variant of $S U F U$ (SUFUvN) was discovered by 3' rapid amplification of cDNA ends (3'RACE) and cDNA clone. SUFUvN contained an additional new protein-coding exon compared with the transcript variant 1 of SUFU (SUFUv1, NM_016169) published in NCBI website. The sequence of the new protein-coding exon was the same as a fragment of intron between exon 10 and 11 of SUFUv1. Thus, an exon skipping occurred in transcription of SUFUv1. Compared with the expression vector of SUFUvN transfected PDAC cells, the corresponding protein expression encoded by SUFUvN (SUFU isoform $\mathrm{N}$ ) was detected in PDAC tissue. Furthermore, it was observed that elevated SUFUvN transcription level was related with lymph node metastasis in PDAC tissues, while neither SUFUv1 nor transcript variant 2 of SUFU (SUFUv2, NM_001178133) did. Our data indicate that there exists a novel transcript variant of SUFU which can be transcribed and translated into corresponding protein and its transcription is related with metastasis of lymph nodes in PDAC.
\end{abstract}

\section{Introduction}

Pancreatic ductal adenocarcinoma (PDAC), also known as pancreatic cancer is one of the most fatal diseases, and the overall 5-year survival rate among patients with pancreatic

Correspondence to: Professor Jun Gao or Professor Zhaoshen Li, Department of Gastroenterology, Shanghai Changhai Hospital, Second Military Medical University, 168 Changhai Road, Shanghai 200433, P.R. China

E-mail: 13816012151@163.com

E-mail: zhsli@81890.net

Key words: suppressor of fused, alternative splicing, pancreatic ductal adenocarcinoma, lymph node metastasis cancer is $<5 \%$. In pancreatic cancer, $90 \%$ of tumors have activating mutations in the KRAS2 oncogene (1). In addition, activation of hedgehog signaling pathway is vital in tumor development. Activity of hedgehog signaling pathway is gradually increased in the progress from pancreatic intraepithelial neoplasia (PanIN) to PDAC (2-4). Mutations of components lead to activation of hedgehog signaling pathway in some types of tumors (5-8). However, no mutations have been detected in components of this pathway in pancreatic cancer. SUFU is an essential negative regulator of mammalian hedgehog signaling pathway. Kasai et al (9) provided evidence that physical binding inhibition of GLI1 by SUFU is dismissed by tethering of SUFU in the cytoplasm of PDAC cells by overexpressed SCL/TAL1 interrupting locus (SIL) and KRAS mutation. However, Chen et al (10) provided convincing data indicating that mouse Sufu sequesters Gli2/3 protein in the cytoplasm and protects them from Spop-mediated protein degradation, providing a Gli protein pool for the production of Gli2/3 activators and repressors. Sufu is necessary for maximal activation of hedgehog pathway. Thus Sufu has an unexpected positive role in controlling mammalian hedgehog signaling. Thus, we proceeded to clarify the function of SUFU in human pancreatic cancer. There are two transcript variants of $S U F U$ published in NCBI website: transcript variant 1 of SUFU (SUFUv1, NM_016169) and transcript variant 2 of SUFU (SUFUv2, NM_001178133). We started by making cDNA clones of alternative splicing transcript variants of $S U F U$ gene in human pancreatic cancer cells.

\section{Materials and methods}

Cell culture and RNA extraction. Seven pancreatic cancer cell lines SW1990, PATU8988, AsPC-1, BxPC-3, CFPAC-1, Capan-2, PANC-1 were cultured in DMEM medium supplemented with $10 \%$ FBS and incubated in a $5 \% \mathrm{CO}_{2}$ humidified incubator at $37^{\circ} \mathrm{C}$. Total RNA was extracted from cells using RNA iso Plus (Takara, Japan) according to the manufacturer's instructions. The yield of RNA was determined using a spectrophotometer (NanoDrop 2000, Thermo Scientific, USA) and the integrity was evaluated using agarose gel electrophoresis stained with ethidium bromide.

$3^{\prime}$ rapid amplification of cDNA ends (3'RACE) and nested $P C R$. Two reference sequence transcript variants of $S U F U$ 
Table I. Primers used in 3'RACE and RT-PCR of SUFUvN 5'cDNA.

\begin{tabular}{ll}
\hline Primers & \multicolumn{1}{c}{ Sequences } \\
\hline $\begin{array}{l}\text { First cycle of } \\
\text { nested PCR }\end{array}$ & \\
P1 & 5'-CGC AAA GAC AGC CTG GAAAGT GAC-3' \\
3'RACE & 5'-TACCGTCGTTCCACTAGTGATTT-3' \\
outer primer & \\
Second cycle & \\
of nested PCR & \\
P2 & 5'-TCC TGC ATG GAC GGC ACT TTA C-3' \\
3'RACE & 5'CGCGGATCCTCCACTAGTGATTTCAC \\
inner primer & TATAGG-3' \\
RT & \\
SUFU ORF & 5'-CTAGTGTAGCGGACTGTCGAACA-3' \\
reverse trans- & \\
cription primer & \\
PCR & \\
P3 & 5'-CTCCAGGTTACCGCTATCG TC-3' \\
P4 & 5'-TTCGGTCAACAGAATCAGGTTTC-3' \\
\hline
\end{tabular}

gene are published on NCBI. They are transcript variant 1 of SUFU (SUFUv1, NM_016169) and transcript variant 2 of SUFU (SUFUv2, NM_001178133). 3'RACE was made to obtain the 3' cDNA of SUFU transcripts.

3'-Full RACE Core Set Ver.2.0 (product code, D314, Takara) kit contains: 3 'RACE adaptor (a reverse transcription primer adaptor which can addict to mature mRNA poly A tail by its poly $\mathrm{T}$ sequences), M-MLV reverse transcriptase, 3'RACE outer primer (reverse primer of the first nested PCR), 3'RACE inner primer (reverse primer of the second nested PCR), etc. The steps are shown in Fig. 1A.

We performed reverse transcription. The reaction consisted of $1 \mu \mathrm{l}$ total RNA (500 ng/ $\mu \mathrm{l}), 1 \mu \mathrm{l}$ 3'RACE adaptor, $0.25 \mu \mathrm{l}$ M-MLV reverse transcriptase, $0.25 \mu 1$ RNase inhibitor, $2 \mu 1$ 5X M-MLV buffer, $1 \mu \mathrm{l}$ dNTP mixture and $4.5 \mu 1$ RNase-free $\mathrm{dH}_{2} \mathrm{O}$, in a total volume of $10 \mu \mathrm{l}$. Reaction was performed in a GeneAmp ${ }^{\circledR}$ PCR System 9700 (Applied Biosystems, USA) for $60 \mathrm{~min}$ at $42^{\circ} \mathrm{C}$, followed by heat inactivation of RT for $15 \mathrm{~min}$ at $70^{\circ} \mathrm{C}$.

First cycle of nested PCR. The forward primer of the first nested PCR was designed by us according to the kit instructions (Table I). We call this forward primer P1. It is situated in the 9th exon of SUFUv1. The first base pair of P1 is situated in the $1,218 \mathrm{bp}$ of the whole RNA sequences. The reverse primer of the first nested PCR is in the kit called 3'RACE outer primer. The reaction consisted of $3 \mu 1$ reverse transcription product, $7 \mu \mathrm{l} 1 \mathrm{X}$ cDNA dilution buffer, $2 \mu \mathrm{l} \mathrm{P} 1$ (10 $\mu \mathrm{mol} / \mathrm{l})$, $2 \mu 1$ 3'RACE outer primer, $5 \mu 1$ 10X Ex Taq buffer (including $\left.\mathrm{Mg}_{2}\right), 0.25 \mu \mathrm{l}$ Ex Taq enzyme and $30.75 \mu \mathrm{l} \mathrm{dH}_{2} \mathrm{O}$. Reactions were performed in a GeneAmp PCR System 9700 for 3 min at $94^{\circ} \mathrm{C}$, followed by 25 cycles of $94^{\circ} \mathrm{C}$ for $30 \mathrm{sec}, 55^{\circ} \mathrm{C}$ for $30 \mathrm{sec}$, $72^{\circ} \mathrm{C}$ for $5 \mathrm{~min}$, and a last step of $72^{\circ} \mathrm{C}$ for $10 \mathrm{~min}$.

Second cycle of nested PCR. The forward primer of the second nested PCR was also designed by us according to the kit instruction (Table I). We call this forward primer P2. It is situated in the 10th exon of SUFUv1. The first base pair of P2 is situated in the $1,357 \mathrm{bp}$ of the whole RNA sequences. The reverse primer of the second nested PCR is in the kit called 3'RACE inner primer. The reaction consisted of $1 \mu \mathrm{l}$ the first nested PCR product, $8 \mu 1 \mathrm{dNTP}$ mixture, $5 \mu 110 \mathrm{X}$ Ex Taq buffer (including $\left.\mathrm{Mg}_{2}\right), 0.5 \mu \mathrm{l} \mathrm{Ex}$ Taq enzyme, $2 \mu \mathrm{l} 2$ (10 $\left.\mu \mathrm{mol} / \mathrm{l}\right)$,

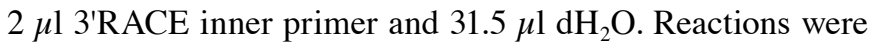
performed in a GeneAmp PCR System 9700 for $3 \mathrm{~min}$ at $94^{\circ} \mathrm{C}$, followed by 30 cycles of $94^{\circ} \mathrm{C}$ for $30 \mathrm{sec}, 55^{\circ} \mathrm{C}$ for $30 \mathrm{sec}, 72^{\circ} \mathrm{C}$ for $5 \mathrm{~min}$, and a last step of $72^{\circ} \mathrm{C}$ for $10 \mathrm{~min}$.

The product of second nested PCR was ligated with pMD-18T vector, and infected DH5 $\alpha$. Then, we selected 10 positive clones, and made sequence detection.

RT-PCR to identify the 5' cDNA sequence of novel transcript variant of SUFU (SUFUvN). We performed reverse transcription by SUFU ORF reverse transcription primer (Table I), then, by PCR. The forward primer of PCR was designed by us called P3 (Table I). It is situated in the first exon of SUFUv1. Reverse primer of PCR was also designed by us called P4 (Table I). It contains partial 11th exon of SUFUv1 and partial new exon. Product of PCR was used for sequence detection. The steps are shown in Fig. 1B.

Construction of expression clones of SUFUvN and SUFUv1. The forward primer terminal is added with protection base pairs, BamHI enzyme sites and Kozak sequences. The reverse primer terminal is added with protection base pairs, AgeI enzyme sites. The forwad primer and reverse primer were designed to clone open reading frame (ORF) of $S U F U$. We performed reverse transcription by SUFU ORF reverse transcription primer (Table I), then by PCR. The sequence of forward primer is: 5'-cgggatccGGGATGGC GGAGCTGCGGCCTA-3'. The sequence of reverse primer is: 5'-gegcaccggtCTAGTGTAG CGGACTGTCGAACA-3'. $B a m \mathrm{HI}$ and AgeI double enzyme digestion was made in both PCR product and pcDNA3.1mychisA (+) expression vector. After ligation of PCR product with pcDNA3.1mychisA (+) expression vector and DH5 $\alpha$ infection we selected positive clones and sequence detection was performed.

Cell transfection. SW1990 cells were transiently transfected with empty vector, SUFUv1 and SUFUvN expression vector, respectively, by use of Lipofectamine 2000 kit (Takara) according to the manufacturer's instructions. The cells were cultured for $24 \mathrm{~h}$ after transfection. Then the cells were harvested for further studies.

Western blotting. The empty vector, SUFUv1 and SUFUvN expression vector transfected SW1990 cells were collected. Cells were dissolved in cell lysis buffer. Protein of pancreatic cancer tissue was extracted by RIPA lysis buffer. The lysates were cooled with ice for $30 \mathrm{~min}$, and then centrifuged at $13,000 \mathrm{x}$ g for $30 \mathrm{~min}$. Proteins $(10 \mu \mathrm{g})$ in the collected supernatant were separated by SDS-PAGE on $12 \%$ gels and then transferred to PVDF membranes. The membrane, after a block with 5\% skim milk, was incubated with primary antibodies to SUFU (rabbit anti-human monoclonal antibody, catalog no. NBP1-40515, Novus, USA) and GAPDH (ab97626, 
A

Target mRNA

5 ,

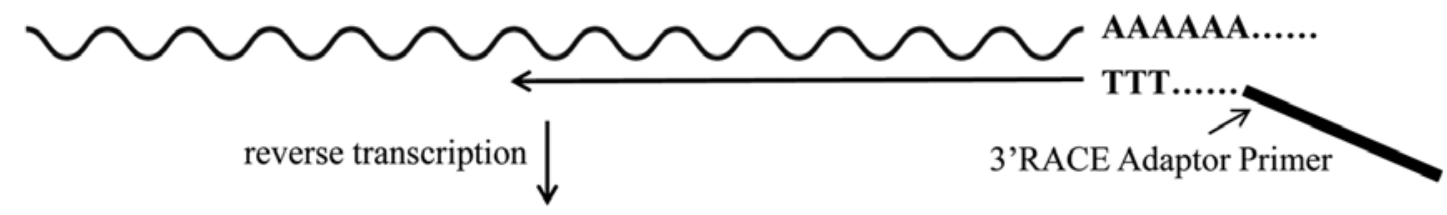

5 ,

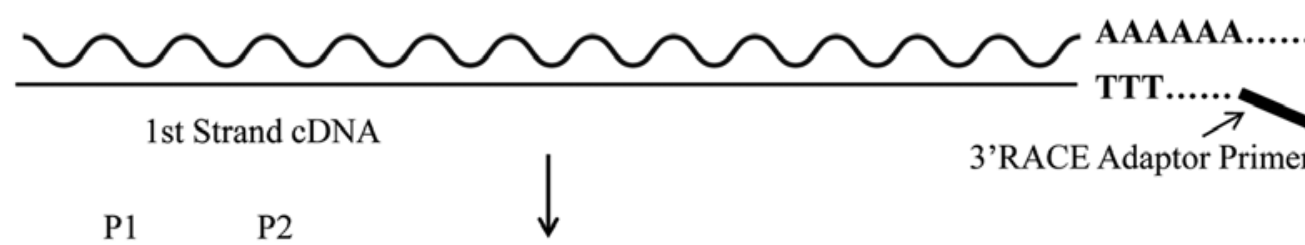

3 ,

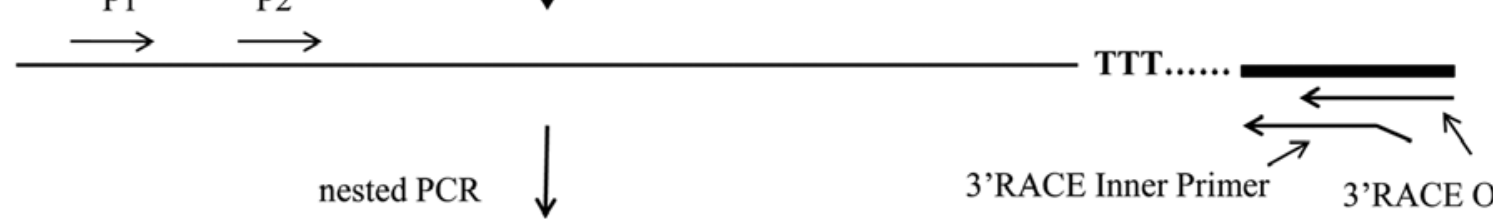

nested PCR $\downarrow$ 3'RACE Inner Primer

double strand cDNA
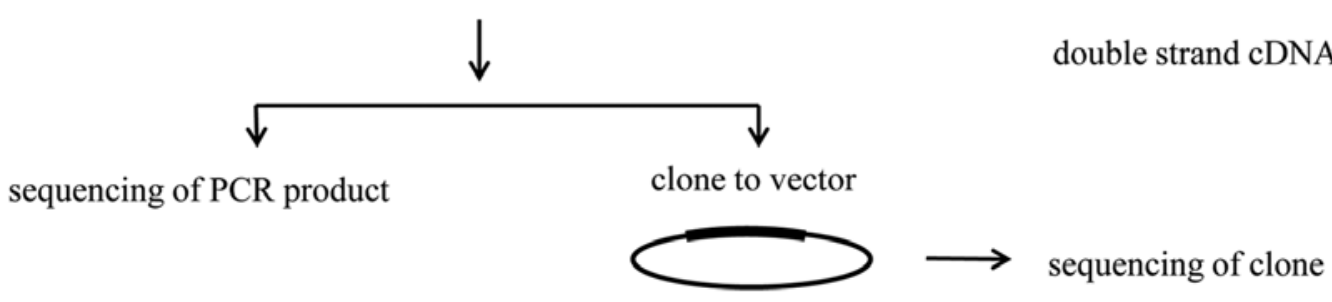

B

5 ,

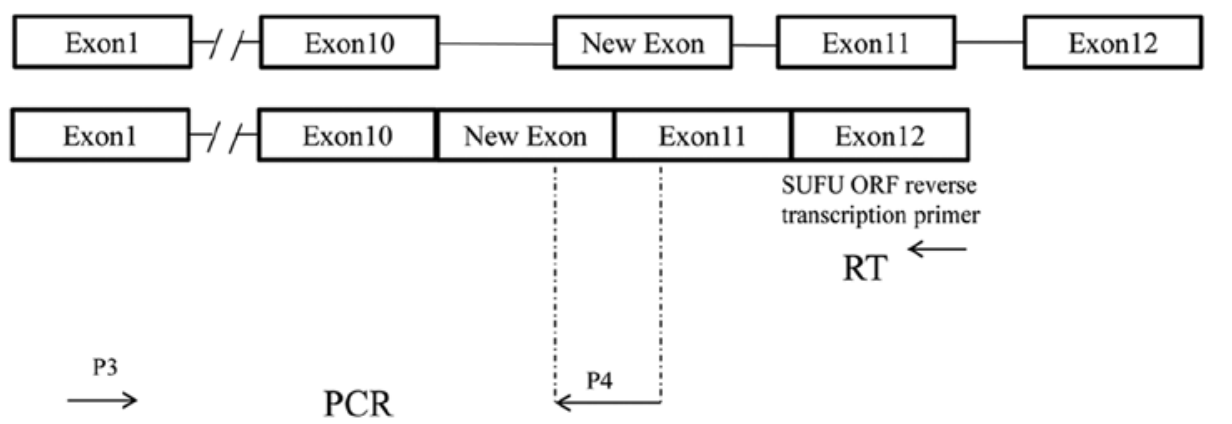

C

\begin{tabular}{|c|c|c|c|}
\hline \multirow[t]{2}{*}{ SUFUvN } & Exon10 & New Exon & Exon11 \\
\hline & \multicolumn{2}{|c|}{ probe } & \\
\hline \multirow{2}{*}{$\begin{array}{c}\text { SUFUv1 } \\
\text { (NM_016169) }\end{array}$} & Exon 10 & Exon11 & Exon 12 \\
\hline & \multicolumn{2}{|c|}{ probe } & \\
\hline $\begin{array}{c}\text { SUFUv2 } \\
\text { (NM_001178133) }\end{array}$ & Exon10 & Exon11, & \\
\hline
\end{tabular}

Figure 1. Schematic diagram. (A) Steps of 3'RACE (3' rapid amplification of cDNA ends). (B) Description of experiment for identifying $5^{\prime}$ cDNA of SUFU novel variant. (C) MGB probe site detecting transcription level of different variants of SUFU.

Abcam, Cambridge, UK). This SUFU antibody is made by $\mathrm{N}$ terminal of SUFU antigen, so it can bind the $\mathrm{N}$ terminal of protein encoded by SUFUv1, SUFUv2 and SUFUvN (SUFU isoform 1, isoform 2 and isoform $\mathrm{N}$ ). Primary antibody is 1:200 diluted to detect SUFU isoforms. The secondary antibody is HRP labeled goat anti-rabbit polyclonal IgG antibody. 
$\mathbf{A}$

Cycle 1

(P1 and 3'RACE Outer Primer)
Cycle 2

(P2 and 3'RACE Inner Primer)

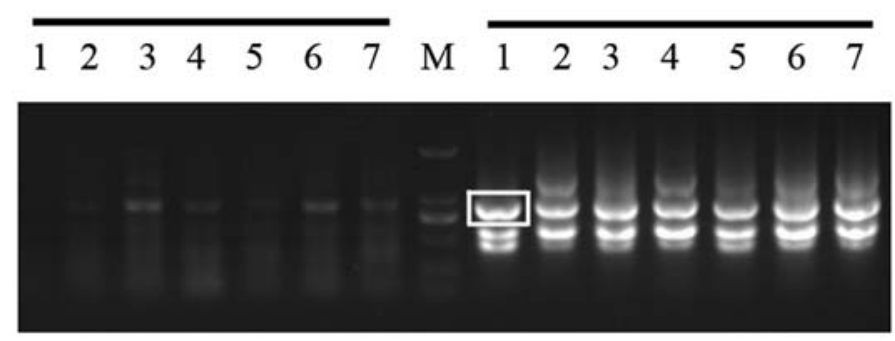

$\mathbf{B}$

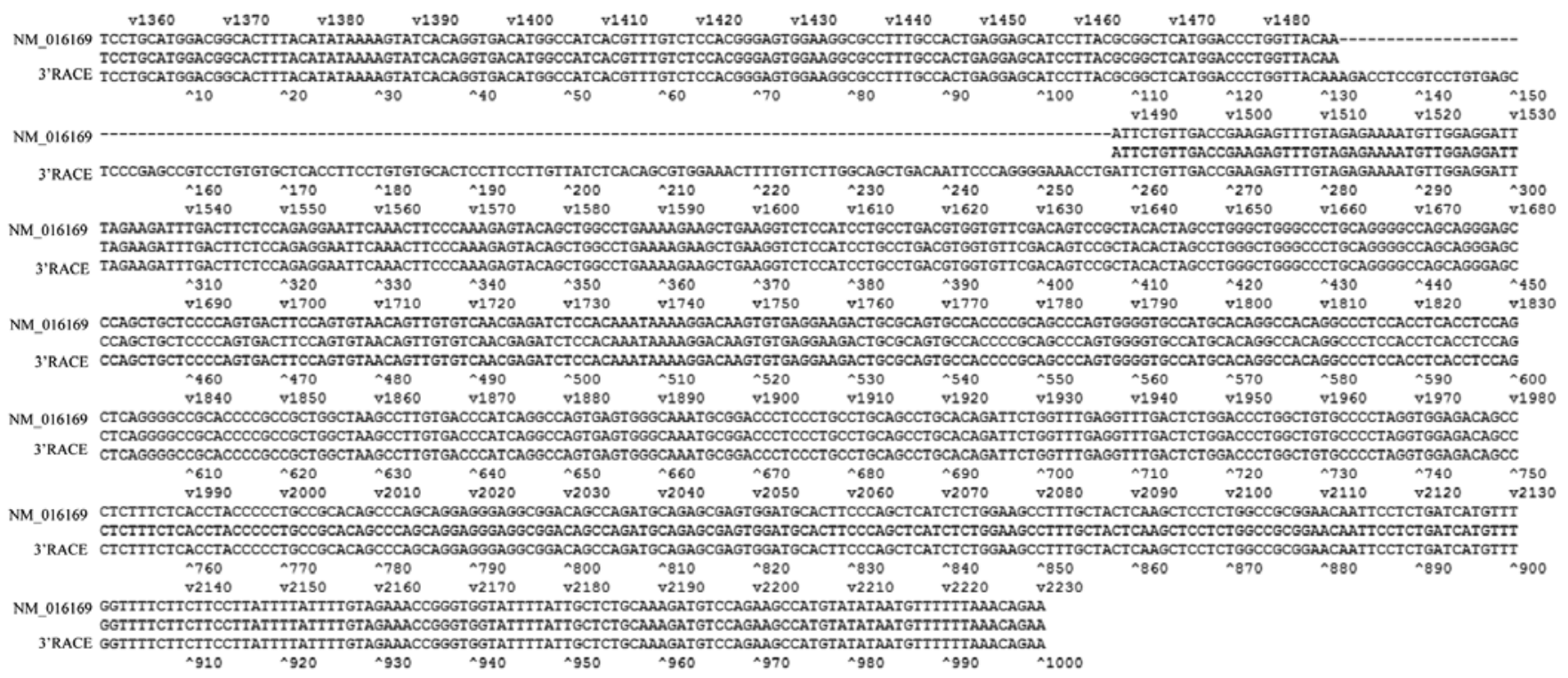

Figure 2. 3'RACE of SUFU. (A) The products of the first cycle and second cycle of 3'RACE nested PCR amplification for SUFU cDNA from pancreatic cancer cells. Lane M, DNA marker (2,000, 1,000, 750, 500, 250 and 100 bp). Lanes 1-7, SW1990, PATU8988, AsPC-1, BxPC-3, CFPAC-1, Capan-2, PANC-1. The indicated band $\sim 1,000$ bp in SW1990 was cloned and sequenced. (B) Sequence alignment of NM_016169 and 3'RACE clone. The mismatch sequence is the new exon.

GAPDH is used as inner control. ECL kit (product code, 34096, Thermo Fisher Scientific, USA) and the camera are used to capture the results.

Patients, tissue samples and total RNA extraction of tissue samples. Tumors were obtained from 40 adult patients diagnosed with pancreatic ductal adenocarcinoma who undergwent surgery at The Second Military Medical University affiliated Changhai Hospital between 2013 and 2014. This study was approved by the Ethics Committee of Changhai Hospital. The total RNA of the pancreatic cancer tissue was extracted by mirVana ${ }^{\mathrm{TM}}$ miRNA isolation kit (am1561, Takara). DNA contamination was removed by DNase in this kit. Total RNA was stored at $-70^{\circ} \mathrm{C}$.

Real-time quantitative RT-PCR using TaqMan MGB probe method to detect transcription levels of different SUFU variants in PDAC tissues of 40 patients. The $18 \mathrm{~s}$ control (Hs99999901_s1) MGB probe and primers kit were purchased from Invitrogen Co., USA. The primer pairs and the TaqMan MGB probe sequences of SUFU different variants were designed by Primer Express 3.0 software and were synthesized by Invitrogen Co. The sequences are shown in Table II, and the probes are shown in Fig. 1C.
Quantification was performed with a two-step reaction process: reverse transcription (RT) and PCR. Each RT reaction consisted of $1 \mu \mathrm{g}$ RNA, $2 \mu 1$ of PrimerScript buffer, $0.5 \mu \mathrm{l}$ of oligodT, $2 \mu \mathrm{l}$ of random 6 mers and $0.5 \mu \mathrm{l}$ of PrimerScript RT Enzyme Mix I (Takara), in a total volume of $10 \mu \mathrm{l}$. Reactions were performed in a GeneAmp PCR System 9700 (Applied Biosystems) for $15 \mathrm{~min}$ at $37^{\circ} \mathrm{C}$, followed by heat inactivation of RT for $5 \mathrm{sec}$ at $85^{\circ} \mathrm{C}$. Real-time PCR was performed using LightCycler ${ }^{\circledR} 480$ II Real-time PCR Instrument (Roche, Swiss) with $20 \mu \mathrm{l}$ PCR reaction mixture that included $10 \mu \mathrm{l}$ of $2 \mathrm{X}$ TaqMan mix (Takara), $1 \mu \mathrm{l}$ of $20 \mathrm{X}$ probe and primers mixture (Invitrogen), $2 \mu \mathrm{l}$ of cDNA, and $7 \mu \mathrm{l}$ of nuclease-free water. Reactions were incubated in a 384-well optical plate (Roche) at $95^{\circ} \mathrm{C}$ for $10 \mathrm{~min}$, followed by 40 cycles of $95^{\circ} \mathrm{C}$ for $10 \mathrm{sec}, 60^{\circ} \mathrm{C}$ for $30 \mathrm{sec}$. Each sample was run in triplicate for analysis. At the end of the PCR cycles, melting curve analysis was performed to validate the specific generation of the expected PCR product. The expression levels of mRNAs were normalized to 18s rRNA and were calculated using the $2^{-\Delta \Delta \mathrm{Ct}}$ method.

Statistical analyses. The relation of SUFU variant mRNA RQ values $(-\Delta \Delta \mathrm{Ct})$ with clinical characteristics were assessed by Mann-Whitney U test. Statistical analysis was processed by 


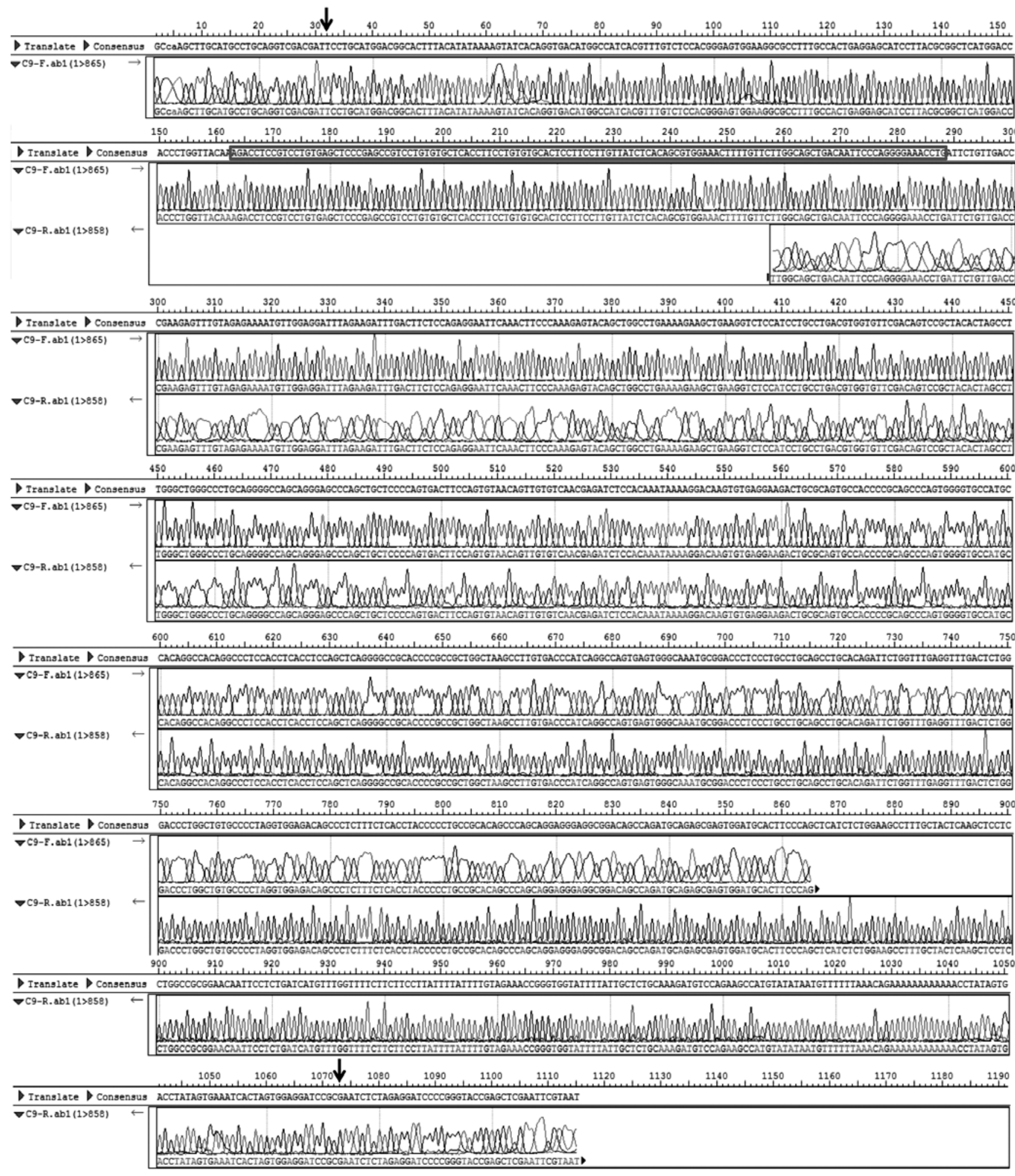

Figure 3. 3'RACE clone sequencing data. This is the direct sequence of clone 9 of the second cycle PCR products. The triangles indicate the insert sites of cloning vector, the frame indicates the new exon.

the SPSS 18.0 software package. P-values $<0.05$ were considered statistically significant.

\section{Results}

Identification of 3'- and 5' cDNA sequences of novel SUFU transcript variant $(S U F U v N)$. Following the strategy described in Fig. 1A, the 3'RACE amplification for 3' cDNA of $S U F U$ in pancreatic cancer cells was performed. The products of first cycle (using P1 and 3'RACE outer primer) and second cycle of nested PCR (using P2 and 3'RACE inner primer) are shown in Fig. 2A. A cDNA fragment of $\sim 1,000$ bp products from SW1990 cells was cloned into pMD18-T and sequenced. As shown in Figs. 2B and 3, a novel cDNA fragment sequence 
Table II. Probes detecting different transcription variants of SUFU.

\begin{tabular}{ll}
\hline Transcript variants & \multicolumn{1}{c}{ Primer pairs and MGB probe } \\
\hline $\begin{array}{l}\text { NM_016169 } \\
\text { Forward primer } \\
\text { Reverse primer } \\
\text { Probe }\end{array}$ & $\begin{array}{l}\text { CACTGAGGAGCATCCTTACGC } \\
\text { AGCTGTACTCTTTGGGAAGTTTGAA } \\
\text { NM_001178133 }\end{array}$ \\
$\begin{array}{l}\text { Forward primer } \\
\text { Reverse primer }\end{array}$ & CCACTGAGGAGCATCCTTACG \\
Probe & FAM-CTGGTTACAACTCTGAACC-MGB \\
Sufu-vN & \\
Forward primer & AGGCGCCTTTGCCACTG \\
Reverse primer & ACACAGGAAGGTGAGCACACAG \\
Probe & FAM-TGGTTACAAAGACCTCCGT-MGB \\
\hline
\end{tabular}

was found at 3 ' termination of $S U F U$ gene compared with cDNA of transcript variant 1 of SUFU (SUFUv1, NM_016169) and transcript variant 2 of SUFU (SUFUv2, NM_001178133) published in NCBI database. This fragment sequence contains a new protein-coding exon (126 bp) the same as a fragment of the intron sequence $(102,625,811-102,625,936$ of the human chromosome 10, hg38 version) between exon 10 and 11 of SUFUv1. We hypothesized that there exists a novel alternative splicing transcript variant of $S U F U$ (SUFUvN) which contains an additional new exon compared with SUFUv1.

In order to identify the full length of SUFUvN cDNA squence, the 5' cDNA fragment of SUFUvN was amplified by RT-PCR following the strategy described in Fig. 1B, in which a specific primer $4(\mathrm{P} 4)$ covering the new exon and exon 11 of SUFUv1 pairing with a specific primer 3 (P3) siting in exon 1 of SUFUv1 were used. As shown in Fig. 4A, a cDNA fragment of 1,400 bp products from SW1990 cells was obtained by RT-PCR and its sequence was the same as the exon 2-exon 10 of SUFUv1 cDNA. The combination of exon 10 and new exon was also been detectable in 1,400 bp product (data not shown). Thus, the new exon is combined with exon 1-10 in 5'-terminal and with exon 11-12 in $3^{\prime}$ terminal.

Clone of full length of SUFUvN. RT-PCR product of SUFU transcript was double enzyme digested by BamHI and AgeI and was ligated with expression vector pcDNA3.1mychisA (+). After infection to DH5 $\alpha$, positive clones were selected and sequenced. From the sequencing and alignment result (data not shown), we identified two kinds of SUFU cDNA clones. They are SUFUv1 and full length of SUFUvN as described above. Figure of BLAT in UCSC web site show us SUFUvN, 5 'cDNA of SUFUvN, 3'cDNA of SUFUvN, and other two SUFU variants (Fig. 4B).

Table III. Relation of SUFU variant transcription level in the pancreatic cancer tissues with clinical characteristics of pancreatic cancer patients.

\begin{tabular}{|c|c|c|c|c|c|c|c|}
\hline \multirow[b]{2}{*}{ Characteristics } & \multirow[b]{2}{*}{$\mathrm{N}$} & \multicolumn{2}{|c|}{ SUFUvN } & \multicolumn{2}{|c|}{ SUFUv1 } & \multicolumn{2}{|c|}{ SUFUv2 } \\
\hline & & $-\Delta \Delta \mathrm{Ct}$ & P-value & $-\Delta \Delta \mathrm{Ct}$ & P-value & $-\Delta \Delta \mathrm{Ct}$ & P-value \\
\hline \multicolumn{8}{|l|}{$\mathrm{N}$ stage } \\
\hline No & & $1.39 \pm 0.91$ & $<0.0001$ & $2.13 \pm 1.04$ & 0.08 & $2.38 \pm 1.49$ & 0.05 \\
\hline N1 & & $5.84 \pm 1.07$ & & $2.80 \pm 0.68$ & & $3.22 \pm 1.11$ & \\
\hline \multicolumn{8}{|l|}{ T stage } \\
\hline $\mathrm{T} 0-2$ & & $2.85 \pm 2.44$ & 0.23 & $2.44 \pm 1.20$ & 0.95 & $2.38 \pm 1.47$ & 0.29 \\
\hline $\mathrm{T} 3-4$ & & $3.98 \pm 2.43$ & & $2.48 \pm 0.79$ & & $3.00 \pm 1.29$ & \\
\hline \multicolumn{8}{|l|}{ Age (years) } \\
\hline$<65$ & & $3.56 \pm 2.56$ & 0.86 & $2.40 \pm 1.05$ & 0.64 & $2.97 \pm 1.37$ & 0.16 \\
\hline$\geq 65$ & & $3.77 \pm 2.25$ & & $2.66 \pm 0.37$ & & $2.31 \pm 1.31$ & \\
\hline \multicolumn{8}{|l|}{ Gender } \\
\hline Male & & $3.68 \pm 2.40$ & 0.83 & $2.47 \pm 0.99$ & 1.00 & $2.76 \pm 1.39$ & 0.84 \\
\hline Female & & $3.47 \pm 2.67$ & & $2.47 \pm 0.81$ & & $2.89 \pm 1.38$ & \\
\hline \multicolumn{8}{|l|}{ Tumor size $(\mathrm{cm})$} \\
\hline$<3$ & & $3.18 \pm 2.58$ & 0.80 & $2.81 \pm 0.73$ & 0.22 & $2.51 \pm 1.37$ & 0.55 \\
\hline$\geq 3$ & & $3.76 \pm 2.45$ & & $2.35 \pm 0.97$ & & $2.90 \pm 1.37$ & \\
\hline \multicolumn{8}{|c|}{ Tumor differentiation } \\
\hline Poor & & $3.30 \pm 2.55$ & 0.98 & $2.11 \pm 0.99$ & 0.33 & $2.67 \pm 1.51$ & 0.76 \\
\hline Well & & $3.67 \pm 2.48$ & & $2.53 \pm 0.92$ & & $2.82 \pm 1.36$ & \\
\hline
\end{tabular}

Novel transcription variant of SUFU (SUFUvN), transcription variant 1 of $S U F U$ (SUFUv1, NM_016169), transcription variant 2 of $S U F U$ (SUFUv2, NM_001178133). Statistically significant, $\mathrm{P}<0.05$. 
A

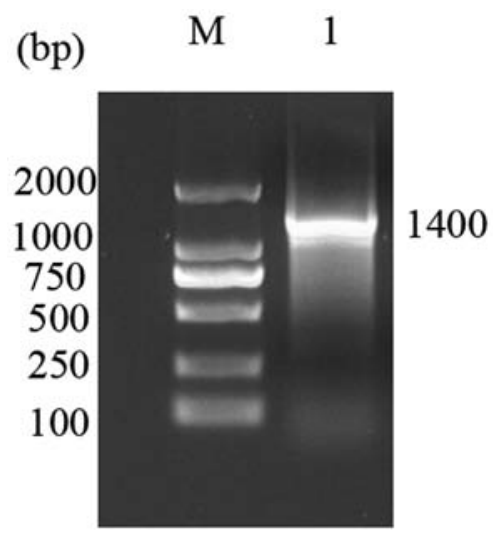

B

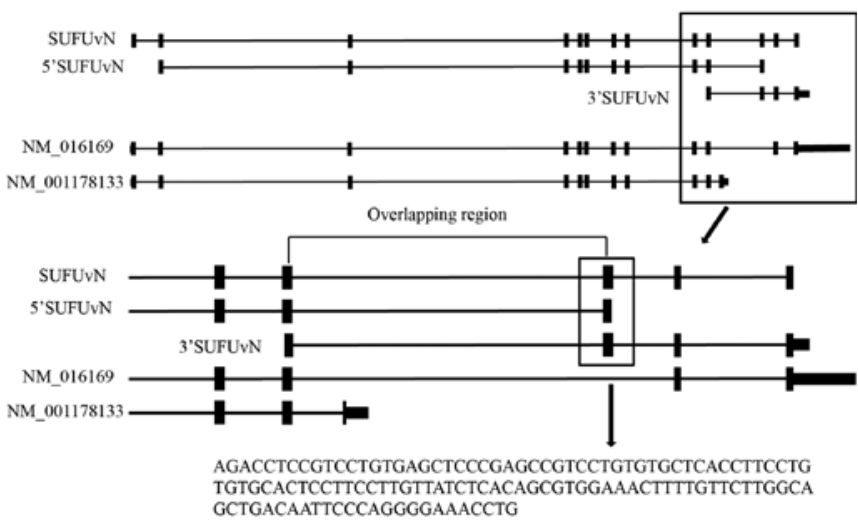

C

SW1990

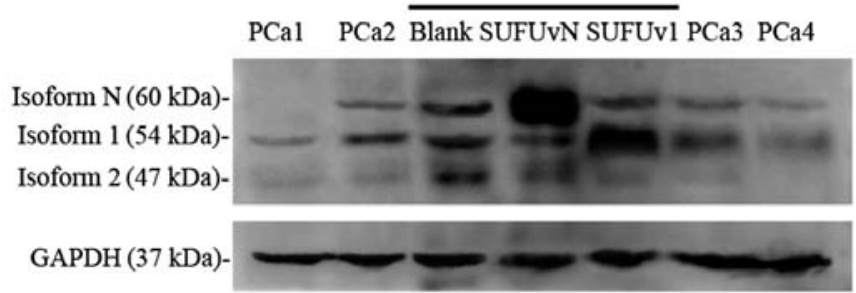

Figure 4. Identification of SUFUvN full length and protein expression. (A) PCR product of 5' cDNA fragment of SUFU novel variant. (B) BLAT figure of SUFUvN cDNA clone. The upper three lines are SUFUvN, 3' and 5 ' cDNA fragment of SUFUvN. The lower two lines are reference sequences of SUFU published in NCBI. The longer one of the reference sequences is NM_016169 (SUFUv1) while the shorter one is NM_001178133 (SUFUv2). (C) Protein expression encoded by SUFUvN was detected by western blotting. Two bands were identified to be protein encoded by SUFUv1 (isoform 1) and isoform $\mathrm{N}$ in PDAC tissues. Isoform 1 is $\sim 54 \mathrm{kDa}$, while isoform $\mathrm{N}$ is $\sim 60 \mathrm{kDa}$. The additional band $\sim 47 \mathrm{kDa}$ may be protein encoded by SUFUv2. GAPDH $\sim 37 \mathrm{kDa}$ is used as inner control. Lanes 1,2,6 and 7, human pancreatic cancer (PCa) tissues. Lane 3, empty vector transfected SW1990 serum. Lane 4, SUFUvN transfected SW1990 serum. Lane 5, SUFUv1 (NM_016169) transfected SW1990 serum. Primary antibody, anti-SUFU and anti-GAPDH.

Identification of protein expression encoded by SUFUvN (isoform $N$ ). There were three bands present in the western blotting figure of PDAC tissue (Fig. 4C). Compared with lanes with SUFUv1 and SUFUvN expression vector transfected SW1990 serum, two bands were identified to be protein encoded by SUFUv1 (isoform 1) and isoform $\mathrm{N}$ in PDAC tissues. Isoform 1 is $\sim 54 \mathrm{kDa}$, while isoform $\mathrm{N}$ is $\sim 60 \mathrm{kDa}$. The additional band $\sim 47 \mathrm{kDa}$ may be protein encoded by SUFUv2 (isoform 2).
A

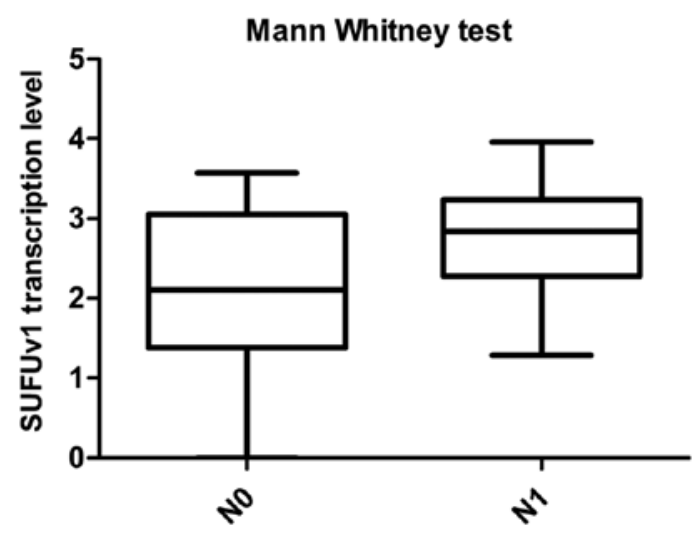

B

Mann Whitney test

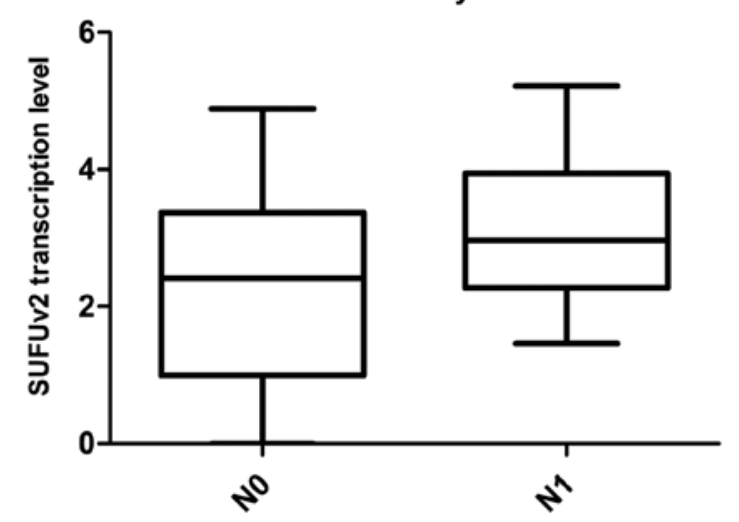

C

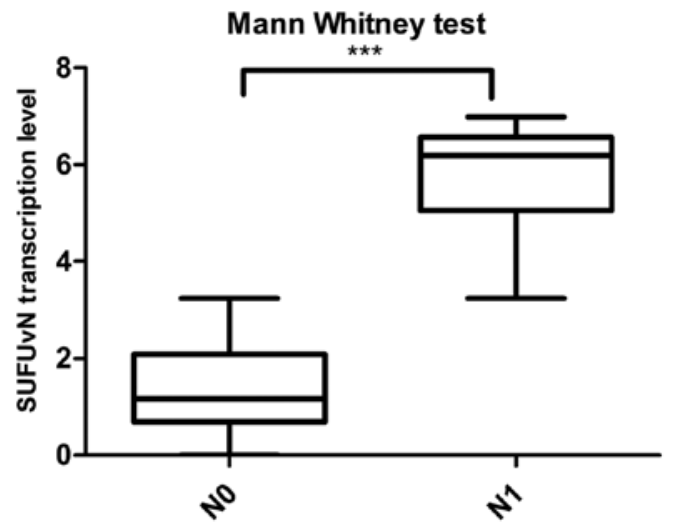

Figure 5. Relationship between transcription of SUFUvN and lymph node metastasis. Transcription levels of SUFUvN were significantly higher in N1 stage than in N0 stage. Transcription levels of SUFUv1 (NM_016169) in N1 and N0 stage show no difference, neither SUFUv2 (NM_001178133). ${ }^{* * *} \mathrm{P}<0.01$.

Detection and clinical relevance of SUFUvN in PDAC tissue. We designed TaqMan MGB probes to identify different transcript variants of SUFU. Each probe is described in the figure, so each probe can uniquely detect the corresponding SUFU variant (Fig. 1C). After detecting transcription levels of SUFU variants in PDAC tissues of 40 patients, we found that SUFUvN transcription levels are significantly higher in PDAC tissues of N1 stage patients than in N0 stage patients, while there is no relationship between other variants transcription levels and lymph node metastasis (Fig. 5). As shown in Table III, we found that the elevated transcription of SUFUvN was related with high $\mathrm{N}$ stage, while no correlation was found with other clinical features, including $\mathrm{T}$ stage, age of onset, gender, tumor size and tumor differentiation. 


\section{Discussion}

Hedgehog (Hh) signaling pathway plays an important role in pathogenesis and development of pancreatic cancer, so investigating this pathway is very important. Mammalian Hh pathway is composed of Hh ligand, target cell receptor patched (Ptch), smoothened (Smo), and transcription factor family Gli. Gli family contains Gli1, Gli2 and Gli3. Gli2 and Gli3 are the targets of primary Hh signaling. When Hh ligand is absent, Gli2/3 undergo proteolysis to generate a transcriptional repressor form. They are processed into active form when Hh ligand is present. Gli1 cannot undergo the same kind of regulated proteolysis and is used exclusively as gene activator. Gli1 is not only a transcription activator but also a transcription target gene of $\mathrm{Hh}$ signaling pathway. Activation of Gli2 and/or Gli3 by Hh often leads to expression of Gli1, which then becomes important in a second wave of activation (11). The formation of either Gli repressor or activator are dependent on primary cilia structure. As a physically binding protein of Gli transcription factor, Sufu is recognized as a negative regulator of hedgehog pathway. However, Chen et al (10) provided convincing data indicating that mouse Sufu regulates Gli2/3 stability by antagonizing the Spop protein of the BTB domain containing protein family. Spop binds to Gli2 and Gli3 and promotes their ubiquitination and degradation in a proteasome-dependent manner. The overexpression of Sufu appears to block Spop-dependent Gli protein reduction. They speculated that Sufu sequesters Gli2/3 protein in the cytoplasm and protects them from Spop-mediated protein degradation, providing a Gli protein pool for the production of Gli2/3 activators and repressors. Sufu is necessary for maximal activation of Hh pathway. Thus Sufu is a positive regulator of Hh signaling pathway in this aspect. Clarification is required to establish whether Sufu is a negative or positive regulator of the Hh pathway. We found a novel alternative splicing transcript of $S U F U$ named SUFUvN by 3 'RACE of $S U F U$ gene and cDNA clone in human pancreatic cancer SW1990 cells. In SUFUvN, a new exon is inserted, which is from intron between 10th and 11th exon of SUFUv1 (NM_016169). The inserted exon makes the carboxyl terminal of protein encoded from SUFUvN (isoform N) different from protein encoded from SUFUv1 (isoform 1) and may alter the function of the expressed protein. A further statistical analysis of clinical data revealed that transcription of SUFUvN is related to metastasis of lymph nodes. A study made by another group gives us some tips on SUFU and GLI interaction (12). The study found the ability of GLI binding of different SUFU isoforms will differ because of their different carboxyl terminal structure. To our knowledge, direct binding of Sufu to all three Gli transcription factors has been well documented (13-17). Sufu binds Gli: the amino and carboxyl terminal of Sufu bind the carboxyl and amino terminal of Gli, respectively. Thus we hypothesize that SUFU functions mentioned in our experiment are loaded by different variants of it according to their affinity to GLI. SUFUvN is possibly a positive regulator of Hh pathway because it has more affinitive to GLI2 and GLI3, while SUFUv1 is more likely to be a negative regulator of Hh pathway because of its affinity to GLI1. So, in pancreatic cancer tissue, endogenous SUFUvN binds with GLI2/3 to antagonize their degradation. High level of endogenous SUFUvN leads to more activation of Hh pathway, and accelerates tumor metastasis.

Research reports describing gene alternative splicing variants and different function of their proteins are limited. It is partially due to the complicated and limited methods in studying splicing variant. In our study, we designed TaqMan MGB probe and primers according to the unique exon of different variants. This can distinguish transcription of different variants. Although the corresponding protein isoforms can be recognized by western blotting because of the different molecular weights, the subcellular situation of different protein isoforms can not be distinguished by immunohistochemistry stain in tissue section unless unique antibodies are produced. Recognition of subcellular situation of different isoforms is very helpful for studing their functions. Additionally, if in situ hybridization of different variant mRNA is required, new technology and method should be applied, for example the LNA probe $(18,19)$. In addition, the crystal and small-angle X-ray scattering structures of full-length human SUFU and its complex with the key SYGHL motif conserved in all GLIs have been reported by Cherry et al (20). It is demonstrated that GLI binding is associated with major conformational changes in SUFU. If alternative splicing transcription variants of $S U F U$ including SUFUvN were to be added to their study in the future, we could improve the understanding of the affinity between SUFU variants and GLIs.

\section{Acknowledgements}

This study was supported by grants from National Natural Science Foundation of China (nos. 81272663 and 81472279 to Jun Gao, no. 30910103911 to Zhaoshen Li), Shanghai Science and Technology Committee Major Project on Basic Research (no. 11441901800 to Jun Gao), and Shanghai Municipal Bureau of Health Research Topic (no. 2012-2015 to Shude Li). The authors also would like to thank Oebiotech Company for gene expression detection systems.

\section{References}

1. Hidalgo M: Pancreatic cancer. N Engl J Med 362: 1605-1617, 2010.

2. Berman DM, Karhadkar SS, Maitra A, Montes De Oca R, Gerstenblith MR, Briggs K, Parker AR, Shimada Y, Eshleman JR, Watkins DN, et al: Widespread requirement for Hedgehog ligand stimulation in growth of digestive tract tumours. Nature 425: 846-851, 2003.

3. Thayer SP, di Magliano MP, Heiser PW, Nielsen CM, Roberts DJ, Lauwers GY, Qi YP, Gysin S, Fernández-del Castillo C, Yajnik V, et al: Hedgehog is an early and late mediator of pancreatic cancer tumorigenesis. Nature 425: 851-856, 2003.

4. Hidalgo $\mathrm{M}$ and Maitra A: The hedgehog pathway and pancreatic cancer. N Engl J Med 361: 2094-2096, 2009.

5. Ingham PW: The patched gene in development and cancer. Curr Opin Genet Dev 8: 88-94, 1998.

6. Reifenberger J, Wolter M, Knobbe CB, Köhler B, Schönicke A, Scharwächter C, Kumar K, Blaschke B, Ruzicka T and Reifenberger G: Somatic mutations in the PTCH, SMOH, SUFUH and TP53 genes in sporadic basal cell carcinomas. Br J Dermatol 152: 43-51, 2005.

7. Taylor MD, Liu L, Raffel C, Hui CC, Mainprize TG, Zhang X, Agatep R, Chiappa S, Gao L, Lowrance A, et al: Mutations in SUFU predispose to medulloblastoma. Nat Genet 31: 306-310, 2002. 
8. Xie J, Murone M, Luoh SM, Ryan A, Gu Q, Zhang C, Bonifas JM Lam CW, Hynes M, Goddard A, et al: Activating smoothened mutations in sporadic basal-cell carcinoma. Nature 391: 90-92, 1998.

9. Kasai K, Inaguma S, Yoneyama A, Yoshikawa K and Ikeda $\mathrm{H}$ : SCL/TAL1 interrupting locus derepresses GLI1 from the negative control of suppressor-of-fused in pancreatic cancer cell. Cancer Res 68: 7723-7729, 2008.

10. Chen MH, Wilson CW, Li YJ, Law KK, Lu CS, Gacayan R, Zhang X, Hui CC and Chuang PT: Cilium-independent regulation of Gli protein function by Sufu in Hedgehog signaling is evolutionarily conserved. Genes Dev 23: 1910-1928, 2009.

11. Østerlund $\mathrm{T}$ and Kogerman P: Hedgehog signalling: How to get from Smo to Ci and Gli. Trends Cell Biol 16: 176-180, 2006.

12. TostarU,FintaC,RahmanMF,ShimokawaT andZaphiropoulosPG: Novel mechanism of action on Hedgehog signaling by a suppressor of fused carboxy terminal variant. PLoS One 7: e37761, 2012.

13. Kogerman P, Grimm T, Kogerman L, Krause D, Undén AB, Sandstedt B, Toftgård R and Zaphiropoulos PG: Mammalian suppressor-of-fused modulates nuclear-cytoplasmic shuttling of Gli-1. Nat Cell Biol 1: 312-319, 1999.

14. Pearse RV II, Collier LS, Scott MP and Tabin CJ: Vertebrate homologs of Drosophila suppressor of fused interact with the gli family of transcriptional regulators. Dev Biol 212: 323-336, 1999.
15. Stone DM, Murone M, Luoh S, Ye W, Armanini MP, Gurney A, Phillips H, Brush J, Goddard A, de Sauvage FJ, et al: Characterization of the human suppressor of fused, a negative regulator of the zinc-finger transcription factor Gli. J Cell Sci 112: 4437-4448, 1999.

16. Dunaeva M, Michelson P, Kogerman P and Toftgard R: Characterization of the physical interaction of Gli proteins with SUFU proteins. J Biol Chem 278: 5116-5122, 2003.

17. Merchant M, Vajdos FF, Ultsch M, Maun HR, Wendt U, Cannon J, Desmarais W, Lazarus RA, de Vos AM and de Sauvage FJ: Suppressor of fused regulates Gli activity through a dual binding mechanism. Mol Cell Biol 24: 8627-8641, 2004.

18. Nuovo GJ, Elton TS, Nana-Sinkam P, Volinia S, Croce CM and Schmittgen TD: A methodology for the combined in situ analyses of the precursor and mature forms of microRNAs and correlation with their putative targets. Nat Protoc 4: 107-115, 2009.

19. Urbanek MO, Nawrocka AU and Krzyzosiak WJ: Small RNA detection by in situ hybridization methods. Int J Mol Sci 16: 13259-13286, 2015.

20. Cherry AL, Finta C, Karlström M, Jin Q, Schwend T, AstorgaWells J, Zubarev RA, Del Campo M, Criswell AR, de Sanctis D, et al: Structural basis of SUFU-GLI interaction in human Hedgehog signalling regulation. Acta Crystallogr D Biol Crystallogr 69: 2563-2579,2013. 